\title{
Binaural Arousal - Sound That Synchronizes the Brain
}

\author{
Branislav R Tanasic \\ People University, Sabac, Serbia
}

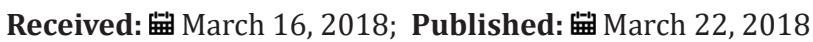

*Corresponding author: Branislav R Tanasic, People University, Koste Abrasevic street 4, Zip code: 15000 Sabac, Sebia, Tel: 381637730 632; Email: tanasicbrana@yahoo.com

\begin{abstract}
Binaural beats are a technology of stereo sound designed to put the brain into the same activity state as when someone are meditating using traditional methods. It is necessary to use a stereo headphone to create a binaural effect in the brain. The pattern is the following- the oscillation discrepancy between the two tones is the frequency at which the binaural beat will pulse. A $250 \mathrm{~Hz}$ tone in the left ear and a $256 \mathrm{~Hz}$ tone in the right ear will produce a binaural beating frequency of $6 \mathrm{~Hz}$, (the middle of the brain`s theta patterns range), only perceptible into the listener's brain. This is important to mention because of the human ear's range of hearing does not go much below $20 \mathrm{~Hz}$, direct listening to such frequencies usually will not register in the listener's perception, especially within older people. Binaural arousal help create a synchronicity between the brain's hemispheres, better cognitive function and higher mental performance.
\end{abstract}

\section{Introduction}

Many cultures throughout history used binaural beats in the rituals with consistent rhythmic sound created extremely powerful healing and spiritual benefits. Some healing sound recordings are based around the healing power of chant and mantra, and are typically unedited/unprocessed recordings of traditional vocal chanting techniques from India, Tibet or other monastic areas. Others use a combination of environmental and/or instrumental sounds combined with synthesized binaural beat frequencies designed to induce specific brainwave states [1]. In his book Earth Magic, Steven Farmer quotes Melinda Maxfield's abstract entitled Effects of Rhytmic Drumming on EEG and Subjective: The use of the drum by indigenous cultures in ritual and ceremony has specific neurophysiological effects and the ability to elicit temporary changes in brain wave activity, and thereby facilitates imagery and possible entry into ASC (alerted state of consciousness), especially the SSC (shamanic state of consciousness), Farmer, 2009.

Heinrich Wilhelm Dove discovered binaural beats 1893, but at the time he didn't have the appropriate devices to scientifically prove his discovery. The basic idea is, when two tuning forks of slightly different pitch are struck simultaneously, the difference between the two pitches the brain perceived as a binaural beat. In other words, he found a way to place very low frequencies directly into the brain. Almost century and a half later, Gerald Oster explains this sound phenomenon: "Slow modulations called binaural beats are perceived when tones of different frequency are presented separately to each ear. The sensation may show certain sounds are processed by the brain" [2].

\section{Brainwave Entrainment}

In the broadest sense, synchronization is the process where two or more systems interact with each other and come to move together. The earliest known scientific discussion of synchronization dates back to 1657 when Christian Huygens built the first pendulum clock. He set them each in motion and found that when he returned the next day, the sway of their pendulums had all synchronized (Pantaleone, 2002: 992). Robert Monroe's research into binaural beats at the Monroe Institute of Applied Sciences confirmed their effectiveness in brain wave entrainment. Monroe conducted thousands of experiments using an EEG machine to monitor subjects' brainwave patterns. The right and left cortical hemispheres generate brain-wave frequencies that are different, one hemisphere is often more active. He also noted that the alterations of brainwave activity occur in the entire brain resonated, the waveforms of both hemispheres becoming identical in frequency, amplitude, phase, and coherence. This is the description of the phenomenon termed hemispheric synchronization [1]. 
A lot of effective substitution of binaural stimuli is Harmonic Box X method, developed by James Mann, author of a guide to programming sound and light entrainment sessions entitled Awakening Mind I. This technique involves two layers of binaural beat producing sine waves that is two frequencies on the left channel and two on the right, all within a close range of each other. For example, $320 \mathrm{~Hz}$ on the left channel and $330 \mathrm{~Hz}$ on the right channel, plus $325 \mathrm{~Hz}$ on the left and $335 \mathrm{~Hz}$ on the right, would both produce a $10 \mathrm{~Hz}$ binaural beat. Additionally, the $320 \mathrm{~Hz}$ on the left channel and the $335 \mathrm{~Hz}$ on the right would produce a $15 \mathrm{~Hz}$ binaural beat, and the $325 \mathrm{~Hz}$ on the left and $330 \mathrm{~Hz}$ on the right would produce a $5 \mathrm{~Hz}$ binaural beat. This example creates entraining frequencies in the Beta, Alpha and Theta ranges [3].

Why is this important? Reminds it, Theta brain waves emit in the range from $4 \mathrm{~Hz}$ to $8 \mathrm{~Hz}$, Alpha waves range from $8 \mathrm{~Hz}$ to $12 \mathrm{~Hz}$ and Beta waves are from $12 \mathrm{~Hz}$ to $30 \mathrm{~Hz}$ [4]. In this way, two layers of the binaural stimulus cover the whole brain which emphasizes the positive effects of synchronism between the brain`s hemispheres, and higher cognitive performance. The experiment carried out by Nantawachara \& Jodchanan [5] showed that the pattern recorded for $10 \mathrm{~min}$ of exposure appeared to be brain functions of a meditative state. Moreover, tension factor of BRUMS was decreased in experimental group compared to control group which resembled the meditation effect. Thus, a 6-Hz binaural beat on a $250 \mathrm{~Hz}$ carrier tone was suggested as a stimulus for inducing a meditative state [5].

An experiment designed and implemented by Kasprzak [5], unambiguously shows significant changes in the spectral density of all ranges: "It was also noted that during the binaural beats exposition, there occurs a statistically significant fall of average amplitudes of a spectral density function of EEG strength signal for alpha $(p<0.001)$ and beta $(p<0.001)$ frequency ranges. For theta $(p<0.0231)$ frequency range, however, there was noted an increase of a spectral density function of EEG strength signal" [6]. Investigation results of the impact of binaural arousal by Lane and associates suggest that the presentation of binaural auditory beats can affect psychomotor performance and mood. This technology may have applications for the control of attention and arousal and the enhancement of human performance $[7,8]$.

\section{Conclusion}

For centuries, people used binaural beats, rhythms with 4.5 strokes per second, and thus caused various mental states. Though without knowing about the binaural beats, Shamans were unmistakably known to cause the shamanic trance, playing the drum around 4 to 7 shots per second for 13 to 15 minutes. As a result of this stimulus, is the brain-wave activity into Theta rhythm? They knew that they recognized the strength and positive effects of binaural stimuli. We live in complex conditions, exposed to stressful situations on a daily basis, so this is an area that must be seriously investigated, especially due to the extremely positive effect of the application of the binaural beats. This is modest attempt through this brief overview, to show the positive effect of binaural stimuli on the functioning of the brain.

\section{References}

1. Gray Tim (2005) The use of sound for control, healing and empowerment, Submitted in partial fulfillment of the requirements for the Degree of Master of Fine Arts in Electronic Music and Recording Media Mills College p. 18

2. Oster Gerald (1973) Auditory Beats in the Brain. Scientific American 229(4): 94-102.

3. Mann James (1998) Awakening Mind I: Creating Sound and Light Sessions on Advanced Programmable Mind Machines. Enlightened Enterprise, Iowa, India.

4. Teplan Michal (2002) Fundamentals of EEG measurement. Measurement Science Review 2(2): 1-11.

5. Nantawachara Jirakittayakorn, Yodchanan Wongsawat (2017) Brain Responses to a 6- z Binaural Beat: Effects on General Theta Rhythm and Frontal Midline Theta Activity. Frontier in Neuroscience 11: 365.

6. Kasprzak C (2011) Influence of Binaural Beats on EEG Signal. Acta Physica Polonica A 119: 990.

7. Lane D James, Kasian J Stefan, Owens E Justin, Marsh R Gail (1998) Binaural auditory beats affect vigilance performance and mood. Physiological Behaviour 63(2): 249-255.

8. Pantaleone James (2002) Synchronization of Metronomes, American Association of Physics Teachers 70(10): 992.

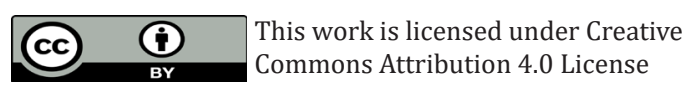

To Submit Your Article Click Here: $\quad$ Submit Article

DOI: 10.32474/OAJBEB.2018.01.000125

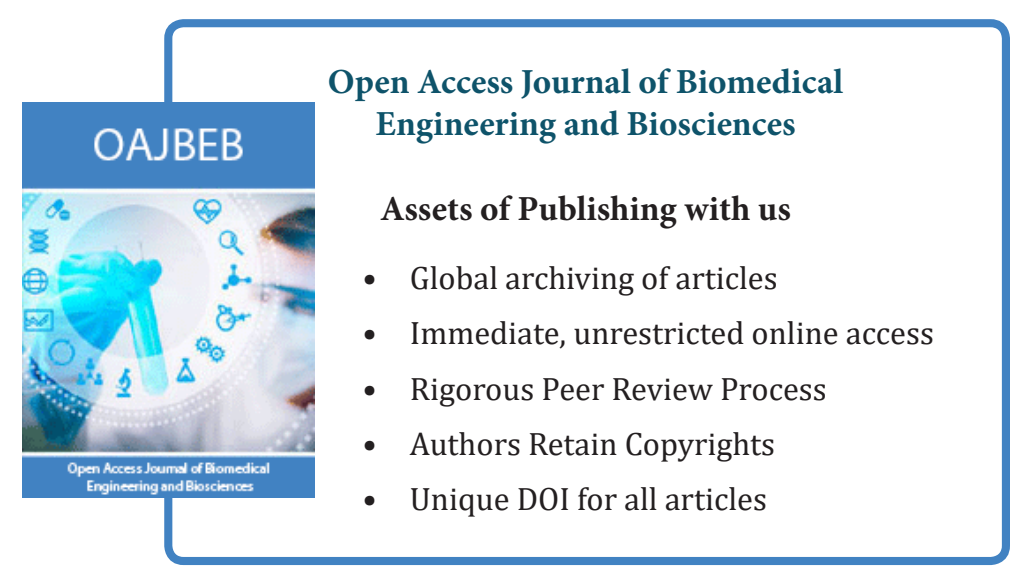

